\title{
Relação entre o estresse e a autoestima de gestantes durante o pré-natal
}

\author{
Relationship between stress and self-esteem of pregnant women during \\ prenatal care
}

Franco Celso da Silva Gomes ${ }^{1}$ (D), Francisca Bruna Arruda Aragão² (D), Liana Linhares Lima Serra ${ }^{3}$ (D), Maria Bethânia Costa Chein ${ }^{1}$ (D), Joelmara Pereira Furtado dos Santos ${ }^{4}$ (D), Liane Maria Rodrigues dos Santos ${ }^{5}$ (D), Andréa Dias Reis ${ }^{6}$ (D), Fernanda Ferreira Lopes ${ }^{1}$ (D),

\section{RESUMO}

Objetivo: analisar os componentes do perfil psicossocial de gestantes durante o pré-natal. Métodos: estudo quantitativo, analítico, realizado com 160 gestantes, entre 14 e 47 anos de idade, atendidas num Hospital de referência do Maranhão, em 2017. Resultados: a maioria das gestantes tinha entre 19 a 35 anos de idade (48,75\%); realizaram de 0 a 5 consultas de pré-natal $(40,65 \%)$ e eram nulíparas $(43,13 \%)$. A análise de regressão linear evidenciou a influência do estresse devido a problemas financeiros e relacionados à família, os itens que mais explicaram o constructo autoestima foram sentir que não é um fracasso e sentir-se satisfeita consigo mesma; A correlação de Pearson do estresse e autoestima foi negativa e com influência significativamente forte. Conclusão: o estresse dentre os fatores psicossociais é o que mais influencia para alterações no pré-natal provavelmente na autoestima, pois quando relacionado com estresse evidenciou relação negativa.

Palavras-Chave: Gestantes; Impacto Psicossocial; Cuidado Pré-Natal; Estresse Psicológico; Apoio Social.

\begin{abstract}
Objective: Analyzing the components of the psychosocial profile of pregnant women during prenatal care. Methods: a quantitative and analytical study performed with 160 pregnant women between the ages of 14 and 47 assisted at a reference hospital in Maranhão, in 2017. Results: the majority of pregnant women were between 19 and 35 years of age (48.75\%); attended to 0-5 prenatal appointments $(40.65 \%)$, and were nulliparous (43.13\%). The linear regression analysis evidenced the influence of stress due to financial and family-related problems; the items that most explained the construct of self-esteem were feeling that they are not a failure and feeling satisfied with themselves; Pearson's correlation of stress and self-esteem was negative and had a significantly strong influence. Conclusion: the stress among the psychosocial factors is the one that most influences prenatal changes probably in self-esteem because when related to stress it showed a negative relation.
\end{abstract}

Keywords: Pregnant Women; Psychosocial Impact; Prenatal Care; Stress, Psychological; Social Support.

1. Programa de Pós-Graduação em Saúde do Adulto, Universidade Federal do Maranhão (UFMA), São Luís (MA), Brasil.

2. Programa de Pós-Graduação Inter Unidades, Escola de Enfermagem de Ribeirão Preto (EERP), Ribeirão Preto (SP), Brasil.

3. Departamento de Ontologia, UFMA, São Luís (MA), Brasil.

4. Mestrado Profissional em Saúde da Família, da Rede Nordeste de Formação em Saúde da Família, UFMA, São Luís (MA), Brasil.

5. Departamento de Enfermagem, Faculdade Maurício de Nassau (UNINASSAU), São Luís (MA), Brasil.

6. Universidade Estadual Paulista Júlio de Mesquita Filho (UNESP), São Paulo (SP), Brasil.

$\bowtie$ Franco Celso da Silva Gomes. Universidade Federal do Maranhão. Av. dos Portugueses, 1966. Prédio do Centro de Ciências Biológicas e da Saúde. CEP 65080-805. São Luís (MA), Brasil. fcsilva-gomes@hotmail.com | Recebido em: 11/10/2019 | Aprovado em: 24/01/2020 


\section{INTRODUÇÃO}

Evidencia-se a atenção integral à Saúde da Mulher, no período gravídico-puerperal, como um desafio para a saúde pública, visto que deve-se dispor de acompanhamento adequado à saúde física e psíquica nas três fases do ciclo gestatório: gravidez, parto e puerpério, a fim de proporcionar assistência integral, efetiva e de qualidade ${ }^{1}$. No Brasil, a Atenção Pré-Natal (APN) apesar de ser um direito universal e de fundamental importância para a garantia de desfechos satisfatórios da gestação, a cobertura não se distribui de forma homogênea e as desigualdades sociais contribuem com a incipiência das ações de acesso e promoção da saúde às mulheres no período gestacional².

Destaca-se, no âmbito das demandas vivenciadas pelas gestantes, o impacto gerado pelos fatores psicossociais percebido pelo estresse como fator limitante para manutenção da saúde. $O$ estresse emerge no cotidiano, pode ser identificado nas relações diárias, associado às próprias modificações da gestação e nos diferentes enfrentamentos relacionados aos papéis desempenhados socialmente, com maior sobrecarga e responsabilidade materna no ciclo gestatório. Sabe-se, no entanto, que a rede de apoio constituiu em elemento essencial para aporte, autoestima e fortalecimento do binômio mãe-filho ${ }^{3}$.

Agrava-se ao estresse diário as tensões no entorno da gravidez e parto, além da necessidade de planejamento que envolve as tarefas de cuidado do bebê e dificuldade para realizar as atividades laborais, domésticas e sociais. Destaca-se, dentre os aspectos psicosociais que nos países desensolvidos, uma em cada dez mulheres sofre de depressão perinatal; enquanto nos países em desenvolvimento, uma em cada cinco mulheres apresenta depressão, o que corrobora com a emergência na oferta de atenção integral, sobretudo às mulheres em situação de vulnerabilidade social ${ }^{4}$.

Salienta-se a importância do apoio da participação paterna na APN no processo saúde-doença-cuidado, considerando as demandas biopsicossociais, a fim de proporcionar responsabilização e inclusão parterna nos cuidados, pois dessa forma a gestante pode ser protegida contra situações adversas à gestação e irá proporcionar maior vínculo e suporte emocional ${ }^{5}$.
Acredita-se que os cuidados do enfermeiro aliado à equipe multiprofissional é capaz de proporcionar assistência qualificada com ênfase nos aspectos biopsicossociais que influenciam a saúde física e psíquica materna. Diante dessas evidências, a assistência aos fatores psicossociais que interferem na gestação das mulheres configuraram fatores motivacionais para a realização desse estudo. Diante da importância do apoio que as mesmas devem receber para garantir uma gestação saudável e tranquila, o presente trabalho tem como objetivo analisar os componentes do perfil psicossocial de gestantes durante o pré-natal.

\section{MÉTODO}

\section{Desenho, Amostra e Aspectos Éticos}

Trata-se de um estudo transversal realizado no período de março a outubro de 2017, no Ambulatório de Obstetrícia da Unidade Materno-Infantil do Hospital Universitário da Universidade Federal do Maranhão (HUUFMA), no município de São Luís - MA, com gestantes que estavam em acompanhamento Pré-Natal.

A coleta de dados ocorreu no período presentado com a população de gestantes em acompanhamento pré-natal no Ambulatório de Obstetrícia. No período da coleta de dados a população era de 450 gestantes. A amostra estudada foi analisada de forma probabilística do tipo aleatória simples, composta por 160 gestantes estabelecida através de cálculo amostral para estudos descritivos com erro amostral de $5 \%$, nível de confiança de $95 \%$ e poder do teste de $80 \% 5$. Foram incluídas mulheres sem limites quanto à idade e período gestacional. Foram excluídas aquelas que só realizaram uma consulta ou que não tinham prontuário no referido hospital.

Todos os procedimentos que envolvem seres humanos foram preservados, conforme preconizado nas "Diretrizes e Normas Regulamentadoras de Pesquisa Envolvendo Seres Humanos" do Conselho Nacional de Saúde n0466/2012. A pesquisa foi apreciada e aprovada pela Comissão Científica do Hospital Universitário (COMIC-HUUFMA), como também pelo Comitê de Ética em Pesquisa (CEP- HUUFMA), com o número: 1.548.766. 


\section{Desfechos do estudo}

$O$ instrumento de pesquisa de dados usado para realização é o Prenatal Phycosocial Profile (PPP), questionário desenvolvido por Curry, Campbell e Christian, em 1994 e adaptado e validado por Weissheimer em 2007. O PPP aborda dados de identificação, clínicos e obstétricos, além disso, consta de três escalas separadas que medem o estresse, o apoio social e a autoestima ${ }^{4}$. 0 questionário validado foi adaptado e traduzido para língua portuguesa nesta pesquisa, visto que no Brasil não se dispõe de uma ferramenta que atenda ao objetivo da pesquisa ${ }^{4}$. Esse instrumento é indicado para as gestantes com prejuízos sociais e econômicos, uma vez que nesse grupo é que existe maior incidência de desfechos insatisfatórios na gravidez (prematuridade e baixo peso ao nascimento) ${ }^{4}$.

Esse instrumento, o PPP, é uma escala do tipo Likert, com 44 itens baseados em instrumen- tos previamente existentes que medem o estresse, o apoio social e a autoestima e que foram escoIhidos após serem avaliados por suas propriedades psicométricas, utilidade clínica e conceptualização.

\section{Análise estatística}

Os dados foram analisados utilizando os recursos do software SPSS (versão 18.0). Inicialmente foi realizada a estatística descritiva, utilizando medidas de frequência absoluta e relativa. A influência de cada item do constructo (Estresse, Apoio do Companheiro, Apoio de outras pessoas e Autoestima) foi analisada por meio da Regressão Linear e expressa com o beta e o coeficiente de explicação $\left(R^{2}\right)$. O Coeficiente de Pearson ( $r$ ) foi utilizado para estimar a correlação entre os componentes do questionário PPP/VP. O nível de significância adotado foi de $5 \%$.

\section{RESULTADOS}

\section{Tabela 1}

Distribuição da amostra segundo alguns referentes das gestantes: escolaridade, idade e trimestre gestacional. São Luís, MA, 2017.

\begin{tabular}{|c|c|c|}
\hline Variáveis & Frequência & Porcentagem (\%) \\
\hline \multicolumn{3}{|l|}{ Escolaridade } \\
\hline Analfabeta & 1 & 0,63 \\
\hline Fundamental incompleto & 20 & 12,50 \\
\hline Fundamental completo & 11 & 6,88 \\
\hline Médio incompleto & 41 & 25,63 \\
\hline Médio completo & 66 & 41,25 \\
\hline Superior incompleto & 9 & 5,63 \\
\hline Superior completo & 12 & 7,50 \\
\hline \multicolumn{3}{|l|}{ Idade } \\
\hline$\leq 14$ anos & 8 & 5 \\
\hline 15 a 18 anos & 27 & 16,88 \\
\hline 19 a 35 anos & 78 & 48,75 \\
\hline$>36$ anos & 47 & 29,37 \\
\hline \multicolumn{3}{|l|}{ Trimestre Gestacional } \\
\hline 10 Trimestre & 32 & 20 \\
\hline 20 Trimestre & 54 & 33,75 \\
\hline 30 Trimestre & 74 & 46,25 \\
\hline
\end{tabular}

(Continua...) 
Tabela 1 (Continuação)

\begin{tabular}{lcc}
\hline Variáveis & Frequência & Porcentagem (\%) \\
\hline Classificação quanto ao número de gestações & 96 & 60,00 \\
$\quad$ Primigesta & 64 & 40,00 \\
$\quad$ Multigesta & & 67,52 \\
Quantidade de consulta pré-natais & 108 & 32,48 \\
$\leq 6$ & 52 & 43,13 \\
$\geq 6$ & & 30,63 \\
Paridade & 69 & 26,25 \\
Nulípara & 49 & 42 \\
$\quad$ Primípara & & \\
Multípara & 49 & \\
\hline
\end{tabular}

Ao se analisar a caracterização da amostra evidenciou-se, em sua maioria, que as gestantes tinham ensino médio completo, faixa etária de 19 a 35 anos, estavam no terceiro trimestre da gestação, primigestas, com número de consultas pré-natais inferior a seis e nulíparas gestantes.

Ao fazer uma análise demonstrativa a respeito dos fatores psicossociais das mulheres no período gestacional usou-se a análise de regres- são, que concebe a avaliação de dados amostrais para saber como duas ou mais variáveis estão relacionadas entre si em uma população.

A análise de regressão linear do constructo Estresse (Tabela 2) dos conceitos de influência significativa sobre a pontuação final, evidencia: preocupações financeiras $\left(R^{2}=0,60\right)$, outras preocupações com dinheiro $\left(R^{2}=0,62\right)$ e problemas relacionados a família $\left(R^{2}=0,29\right)$.

\section{Tabela 2}

Regressão linear simples entre as subescalas do instrumento e o construto Estresse, componentes do Prenatal Phycosocial Profile, atribuídos a 160 gestantes. São Luís,MA, 2017.

\begin{tabular}{llcc}
\hline Variável Estresse & $\mathrm{R}^{2}$ & Beta & Valor de $\mathrm{p}$ \\
\hline Preocupações financeiras & 0,60 & 3,08 & $<0,0001$ \\
Outras preocupações com dinheiro & 0,62 & 2,67 & $<0,0001$ \\
Problemas relacionados à família & 0,29 & 2,31 & $<0,0001$ \\
Mudança de endereço (passada ou futura) & 0,14 & 2,16 & $<0,0001$ \\
Perda recente de alguém especial & 0,15 & 1,79 & $<0,0001$ \\
Gestação atual & 0,18 & 1,62 & $<0,0001$ \\
Sofrer violência ou abuso & 0,06 & 2,62 & $=0,0008$ \\
Problemas com álcool e/ou drogas & 0,06 & 2,81 & $=0,0014$ \\
Problemas no trabalho & 0,07 & 1,95 & $=0,0007$ \\
Problemas relacionados aos amigos & 0,08 & 3,33 & $<0,0001$ \\
Sentir-se sobrecarregada & 0,33 & 2,17 & $<0,0001$ \\
\hline
\end{tabular}

Observou-se que todos os conceitos atribuídos na subescala Apoio Social apresentaram influência significativa sobre a pontuação final do construto Apoio do Companheiro, por meio da análise de regressão linear (Tabela 2). As variáveis que mais explicaram o construto Apoio do Com- 
panheiro foram: permitir falar de coisas pessoais $\left(R^{2}=0,70\right)$ e demonstrar que valoriza o que faço por ele $\left(R^{2}=0,69\right)$. Observou-se, também, que as subescalas do construto Apoio de Outras Pessoas influenciaram significativamente na pontuação final $(p<0,0001)$, por meio da regressão linear simples, com destaque para as subescalas Ajuda a sair de situações difíceis $\left(R^{2}=0,69\right)$, Demonstra que valoriza o que você faz por ele $\left(R^{2}=0,68\right)$ e Estará sempre por perto $\left(R^{2}=0,68\right)$.

Todas as variáveis que compõem o construto Autoestima no PPP - VP (Tabela 3) tiveram influência significativa $(p<000,1)$, sendo que a regressão linear simples revelou que o sentimento de que "não" é um fracasso $\left(R^{2}=0,37\right)$ e sentir-se satisfeita consigo mesma $\left(R^{2}=0,31\right)$ foram os que mais explicitaram o referido construto.

\section{Tabela 3}

Regressão linear simples entre as subescalas do instrumento e o construto Apoio do Companheiro e Apoio de Outras Pessoas, componente do Prenatal Phycosocial Profile, atribuídos a 160 gestantes. São Luís, MA, 2017.

\begin{tabular}{|c|c|c|c|}
\hline Apoio do companheiro & $\mathrm{R}^{2}$ & Beta & Valor de $\mathrm{p}$ \\
\hline Compartilha vivências & 0,49 & 5,63 & $<0,0001$ \\
\hline Ajuda a manter o ânimo & 0,55 & 6,53 & $<0,0001$ \\
\hline Ajuda a sair de situações difíceis & 0,68 & 7,30 & $<0,0001$ \\
\hline Interesse em atividades diárias e problemas & 0,63 & 6,60 & $<0,0001$ \\
\hline Sai da rotina para fazer coisas especiais & 0,44 & 4,86 & $<0,0001$ \\
\hline Permite falar de coisas pessoais & 0,70 & 6,84 & $<0,0001$ \\
\hline Demonstra que valoriza o que faço por ele & 0,69 & 7,53 & $<0,0001$ \\
\hline Tolera altos e baixos & 0,56 & 6,90 & $<0,0001$ \\
\hline Leva a sério as preocupações & 0,58 & 6,50 & $<0,0001$ \\
\hline Diz coisas que tornam a situação clara e fácil & 0,59 & 6,71 & $<0,0001$ \\
\hline Estará por perto & 0,61 & 6,88 & $<0,0001$ \\
\hline Apoio de outras pessoas & $\mathrm{R}^{2}$ & Beta & Valor de $\mathrm{p}$ \\
\hline Compartilha vivências & 0,63 & 7,37 & $<0,0001$ \\
\hline Ajuda a manter o ânimo & 0,64 & 7,42 & $<0,0001$ \\
\hline Ajuda a sair de situações difíceis & 0,69 & 7,25 & $<0,0001$ \\
\hline Interesse em atividades diárias e problemas & 0,64 & 6,82 & $<0,0001$ \\
\hline Sai da rotina para fazer coisas especiais & 0,48 & 5,81 & $<0,0001$ \\
\hline Permite falar de coisas pessoais & 0,59 & 6,89 & $<0,0001$ \\
\hline Demonstra que valoriza o que faço por ele & 0,68 & 7,11 & $<0,0001$ \\
\hline Tolera altos e baixos & 0,64 & 7,03 & $<0,0001$ \\
\hline Leva a sério as preocupações & 0,59 & 6,81 & $<0,0001$ \\
\hline Diz coisas que tornam a situação clara e fácil & 0,61 & 6,47 & $<0,0001$ \\
\hline Estará por perto & 0,68 & 6,99 & $<0,0001$ \\
\hline
\end{tabular}

\section{Tabela 4}

Regressão linear simples entre as subescalas do instrumento e o construto Autoestima, componente do Prenatal Phycosocial Profile, atribuídos a 160 gestantes. São Luís, MA, 2017.

\begin{tabular}{lccc}
\hline Variável Autoestima & $\mathrm{R}^{2}$ & Beta & Valor de $\mathrm{p}$ \\
\hline Sente que tem valor ou é igual aos outros & 0,19 & 2,40 & $<0,0001$ \\
Sente que tem um grande número de qualidades & 0,19 & 2,01 & $<0,0001$ \\
\hline
\end{tabular}

(Continua...) 
Tabela 4 (Continuação)

\begin{tabular}{lccc}
\hline Variável Autoestima & $\mathrm{R}^{2}$ & Beta & Valor de $\mathrm{p}$ \\
\hline Sente que é um fracasso & 0,37 & 3,12 & $<0,0001$ \\
Sente que é capaz de fazer as coisas tanto quanto os outros & 0,09 & 1,47 & $<0,0001$ \\
Sente que não tem muito do que se orgulhar & 0,29 & 2,21 & $<0,0001$ \\
Tem um atitude positiva consigo mesma & 0,21 & 2,52 & $<0,0001$ \\
Sente-se satisfeita consigo mesma & 0,31 & 2,89 & $<0,0001$ \\
Quer ter mais respeito por si mesma & 0,09 & 1,46 & $<0,0001$ \\
Sente-se inútil & 0,20 & 2,08 & $<0,0001$ \\
Não se acha boa que chega & 0,22 & 1,85 & $<0,0001$ \\
Tem controle sobre sua vida & 0,13 & 2,10 & $<0,0001$ \\
\hline
\end{tabular}

A Tabela 4 faz uma demonstração da correlação de Pearson entre os constructos dos com- ponentes acima descritos, relatados no pré-natal, conforme se observa a seguir.

\section{Tabela 5}

Coeficiente de Correlação de Pearson ( $r$ ) e sua significância estatística ( $p$ ) entre os construtos componentes do Pré-natal Psychosocial Profile (PPP-VP). São Luís/MA-BR, 2017.

\begin{tabular}{|c|c|c|c|c|}
\hline \multicolumn{5}{|c|}{ Construtos componentes do PPP-VP } \\
\hline \multirow{2}{*}{$\begin{array}{l}\text { Construtos componentes } \\
\text { do PPP-VP }\end{array}$} & Estresse & Apoio do companheiro & Apoio de outras pessoas & Autoestima \\
\hline & $r(p-v a l o r)$ & $r(p-v a l o r)$ & $r(p-v a l o r)$ & $r(p$-valor $)$ \\
\hline Estresse & - & $-0,026(0,743)$ & $-0,040(0,619)$ & $-0,178(0,025 *)$ \\
\hline Apoio do companheiro & $-0,026(0,743)$ & - & $0,067(0,402)$ & $0,034(0,671)$ \\
\hline Apoio de outras pessoas & $-0,040(0,619)$ & $0,067(0,402)$ & - & $0,071(0,373)$ \\
\hline Autoestima & $-0,178(0,025 *)$ & $0,034(0,671)$ & $0,071(0,373)$ & - \\
\hline
\end{tabular}

$r=$ coeficiente de correlação de Pearson *Correlação estatística significante $(P<0,05)$

PPP $=$ Prenatal Phycosocial Profile

VP- Versão em Português

A correlação estresse $x$ autoestima foi a única que manifestou correlação negativa e influência significativa forte $(r=-0,178 / p=0,025)$.

\section{DISCUSSÃO}

O perfil psicossocial de gestantes tem sido analisado de forma muito insidiosa. Este estudo revelou que fatores relacionados ao financeiro e problemas relacionados à família foram os itens que mais influenciaram ao se analisar o constructo estresse. A atual conjuntura econômica do nosso país, período de crise, pode refletir na gestação atual, pois a gestante tem que comple- mentar as necessidades financeiras com outras atividades, fazendo com que se sinta ainda mais sobrecarregada e dificultando ainda mais a gestação atual, predispondo a grávida para muitas situações desconfortáveis, e por vezes, até complicações obstétricas ${ }^{6}$.

Durante o período gravídico, puerperal, surgem vários fatores que influenciam o desencadeamento de estresse na vida de uma mulher, podendo surgir de características físicas, sociais e econômicas. E a gestação da mulher está em total vulnerabilidade, sendo exposta a diversas exigências, caindo em turbulências emocionais, incapacitando-a em algumas vezes de viver seu dia-a-dia de maneira harmoniosa ${ }^{6}$. 
A forma como cada indivíduo percebe o estresse é algo único e inúmeras podem ser as causas que podem chegar a desencadeá-lo nessa fase da vida da mulher, como extremos de idade, carga horária de trabalho diário ou como ela preenche seu tempo durante o dia, os cuidados que ela recebe, o nível de experiência, entre outros ${ }^{7}$. Além disso, as alterações físicas também podem ser agentes estressores, como o aumento das mamas, episódios de enjoos, vertigem e mal-estar geral ${ }^{8}$.

Esse estresse pode desencadear complicações como baixo peso do recém-nascido (RN), abortamento e a aumento da probabilidade da prematuridade, além de alterações neurológicas, imunitariedade e outras patologias tanto para mãe e para o bebê, todas podendo ocorrer no período gravídico. Pesquisa recente demonstra associação entre o número de eventos estressores durante a gravidez e os efeitos causados para a gestante com a precipitação de depressão pós-parto e outros transtornos mentais comuns na gravide $z^{9}$.

Com relação ao apoio social do companheiro e de outras pessoas durante a gestação, o que foi evidenciado é que todos os itens estudados sugestionaram de forma significativa no constructo avaliado. O apoio social é um processo que pode envolver afeto, amor, empatia, respeito, informações, conselhos, auxílio financeiro, tempo dedicado e disponibilização de recursos, bens e serviços e, além disso, pode contribuir para a adaptação à gestação, às vivências e rotinas ${ }^{10}$.

O apoio social ofertado em redes sociais apresenta uma ampla gama de benefícios relacionados aos aspectos físicos, mentais, psicológicos, sociais e emocionais ${ }^{11}$. A rede social de apoio vai muito além da figura da família, envolve também o círculo de amigos e o que diz respeito à fé e religião, sendo de extrema importância o apoio que esses círculos trazem no ciclo gravídico para o bem-estar físico e mental da gestante. Desse modo, o apoio social prestado a essas gestantes promove a formação de um clima menos estressante e mais favorável para uma vivência do ciclo gravídico ${ }^{12}$.

Outro item avaliado foi o constructo autoestima através de 11 itens em que todos influenciaram de forma significativa, porém, os mais explicativos para o fator foram não sentir-se fracassada e a satisfação pessoal. A gestante acaba por experienciar um misto de sentimentos, muitas vezes decorrentes das alterações hormonais que acontecem. Devido ao aumento dos hormônios e todas as mudanças ocasionadas pela gestação, o humor acaba sendo alterado facilmente ${ }^{13}$.

Ao se estabelecer correlação entre os constructos do perfil psicossocial das gestantes, foi evidenciado que quanto maior o estresse menor será a autoestima da gestante e que essa relação é expressivamente forte.

Para muitas mulheres o fato de estar grávida gera uma alegria e orgulho perante aquelas que desejam a gravidez com otimismo, mas em algumas gestantes esse período gera desconforto, principalmente na sua imagem corporal. A assistência pré-natal entra nesse roteiro de auxílio, pois prepara a mulher para receber o bebê com grande alegria e estimula a sua autoestima e autoconfiança, para se viver uma boa gestação, parto e puerpério ${ }^{14}$.

As limitações desta pesquisa corresponde a um estudo transversal realizado em curto período de tempo, em um único Ambulatório de Obstetrícia de um hospital universitário, bem como a dificuldade encontrada na coleta dos dados, em virtude da falta de organização dos mesmos por categorias. Entretanto, nosso estudo contribuiu para melhorar a assistência pré-natal nas Unidades Básicas de Saúde, através da Estratégia Saúde da Família (ESF), como também desenvolver a percepção do profissional de saúde que presta assistência à saúde acerca de fatores não somente biofísicos, mais que englobam aspectos psicossociais das gestantes durante a assistência pré-natal. Portanto, cabe às instâncias públicas e privadas de saúde enfatizar uma assistência às grávidas de forma integral, contemplando todos os aspectos que possam abordar desde objetivos até os mais íntimos aspectos subjetivos.

\section{CONCLUSÃO}

A gestação não se limita apenas aos aspectos fisiológicos, mas a interferências de fatores psicossociais como estresse, apoio social e autoestima. $O$ estresse e a autoestima foram os constructos que apresentaram menores valores nos itens analisados, além disso, ainda observou-se relação negativa entre eles. 
O suporte social ofertado tanto pelo companheiro quanto por outras pessoas foram na maioria dos itens analisados considerados satisfatórios. Sendo assim, faz-se necessário uma atenção especial aos aspectos financeiros durante a gestação, pois, foi o item mais apontado na pesquisa como influenciador no estresse e possivelmente pode estar influenciando na autoestima. Então, um olhar atento a esses aspectos provavelmente facilitaria ainda mais esse período de vulnerabilidade da mulher.

\section{AGRADECIMENTO}

Fundação de Amparo à Pesquisa e ao Desenvolvimento Científico e Tecnológico do Maranhão - FAPEMA.

\section{REFERÊNCIAS}

1. Balsells MMD, Oliveira TMF, Bernardo EBR, Aquino PS, Damasceno AKC, Castro RCMB, et al. Evaluation of prenatal care process for habitual-risk pregnant women. Acta Paul Enferm. 2018;31(3):247-54. doi: http://dx.doi.org/10.1590/1982-0194201800036

2. Luz LA, Aquino R, Medina MG. Evaluation of the quality of Prenatal Care in Brazil. Saúde Debate. 2018;42(especial2):111-26. doi: http://dx.doi.org/10.1590/0103-11042018S208

3. Monteiro PGA, Souza IC, Rodrigues VCC, Sena MM, Costa JS, Lopes TSS, et al. Percepções de mulheres acerca do estresse vivenciado na gestação. Investigação Qualitativa em Saúde [Internet]. 2018 [cited 2019 Mai 14];2(1):1142-9. Available from: https://proceedings. ciaiq.org/index.php/ciaiq2018/article/view/1889

4. Organización Panamericana de La Salud (OPAS). Pensamiento saludable. Manual para la atención psicosocial de la depresión perinatal [Internet], Washington: OPAS, 2015 [cited 2019 Mai 14]. Available from: http://iris. paho.org/xmlui/handle/123456789/28417

5. Ministério da Saúde (BR), Secretaria de Atenção à Saúde, Departamento de Ações Programáticas Estratégicas Coordenação Nacional de Saúde do Homem. Guia do Pré-Natal do Parceiro para Profissionais de Saúde. Brasília, DF:
Ministério da Saúde [Internet]. 2016 [cited 2019 Mai 14]. Available from: http://portalarquivos2.saude.gov.br/ images/pdf/2016/agosto/11/guia_PreNatal.pdf

6. Bós ÂJG. Epi Info $^{\circledR}$ sem mistérios: um manual prático recurso eletrônico - Dados eletrônicos. Porto Alegre: EDIPUCRS; 2012. 211 p.

7. Boarollit $M$ et al. Avaliação do estresse, depressão, e ansiedade em um grande grupo de gestantes cadastradas na estratégia saúde da família no Bairro São Sebastião, Criciúma. Rev Prog Resid Multi Atenção Básica [Internet] 2016. [cited 2019 Mai 14]; 3(1):1-9. Available from:http://periodicos.unesc.net/prmultiprofissional/article/view/3028/2792

8. Vieira BD, Parizotto APAV. (2014). Alterações psicológicas decorrentes do período gravídico. Unoesc \& Ciência ACBS [Internet] 2014. [cited 2019 Mai 14];4(1):79-90. Available from: https://editora.unoesc.edu.br/index.php/ acbs/article/viewFile/2559/pdf.

9. Alvarenga P, Frizzo GB. Stressful Life Events and Women's Mental Health During Pregnancy and Postpartum Period. Paidéia. 2017;27(66):51-9. doi: http://dx.doi. org/10.1590/1982-43272766201707.

10. Cremonese L, Wilhelm LA, Prates LA, Paula CCD, Sehnem GD, Ressel LB. Social support from the perspective of postpartum adolescents. Escola Anna Nery. 2017;21(4):e20170088. doi: http://dx.doi.org/10.1590/ 2177-9465-EAN-2017-0088.

11. Gomes, I. M., Lacerda, M. R., Rodrigues, J. A. P., Camargo, T. B. D., Zatoni, D. C. P., \& Nascimento, V. S. (2016). The support of social network in homecare. Escola Anna Nery. 2016;20(3):e20160062. doi: http://dx.doi.org/10.5935/ 1414-8145.20160062.

12. Monteiro PGA, Souza IC, Rodrigues VCC, Sena MM, Costa JS, Lopes TSS, et al. Percepções de mulheres acerca do estresse vivenciado na gestação. Investigação Qualitativa em Saúde [Internet] 2018. [cited 2019 Mai 14]; 2(1):1142-9. Available from: https://proceedings.ciaiq. org/index.php/ciaiq2018/article/view/1889/1839.

13. Rezende J, Montenegro CAB. Rezende obstetrícia fundamental. Rio de Janeiro: Guanabara Koogan; 2014. 1012p.

14. Tomaschewski-Barlem JG, Bordignon SS, Costa CFS, Costa CO, Barlem ELD. Promovendo a autoestima na gestação: foco no Acolhimento. Enferm. Foco [Internet] 2016. [cited 2019 Mai 14]; 7(2):83-6. Available from: https://pdfs.semanticscholar.org/85fa/6063927b123f4e69816bd37b9c76d862ca79.pdf. 